\title{
Shunt Revision to Ventriculoatrial Shunt Due to Long-Term Abdominal Distension Complicated by Neonatal Necrotizing Enterocolitis and Cerebrospinal Fluid Overproduction after Ventriculoperitoneal Shunt for the Management of Post-Hemorrhagic Hydrocephalus: A Case Report
}

\author{
Kyoung Jae Park, Yong Cheol Lim, Soo Han Yoon \\ Department of Neurosurgery, Ajou University Medical Center, Ajou University School of Medicine, Suwon, Republic
} of Korea

Corresponding author:

Soo Han Yoon

Department of Neurosurgery,

Ajou University Medical Center,

Ajou University School of

Medicine, 164, World cup-ro,

Yeongtong-gu, Suwon 16499,

Republic of Korea

Tel: +82-31-219-5233

Fax: $+82-31-219-5238$

E-mail: ee80@hanmail.net

Received: August 18, 2019

Revised: September 2, 2019

Accepted: September 6, 2019

\begin{abstract}
A cerebrospinal fluid (CSF) shunt is the most common treatment known to date in the treatment of hydrocephalus. A ventriculoperitoneal (VP) shunt is one of the most commonly favored procedures for CSF diversion. We present a case of shunt revision from a VP shunt to a ventriculoatrial (VA) shunt. The patient was diagnosed with germinal matrix hemorrhage with intraventricular hemorrhage shortly after birth with a history of ileostomy surgery for neonatal necrotizing enterocolitis 10 days after birth. The patient began to develop hydrocephalus around 7 weeks after birth and she had VP shunt surgery at 6 months old for post-hemorrhagic hydrocephalus. After the VP shunt surgery, the patient experienced several months of vomiting and constipation along with worsening of the abdominal distension. Distal abdominal catheter externalization was temporarily performed, and 20 to $30 \mathrm{~mL} / \mathrm{kg}$ of CSF was drained daily. Abdominal distension was still not resolved after 2 weeks and VA shunting was performed. The patient was discharged with improved abdominal distension and was followed for 3 years without any complications. We report a relatively rare case of a shunt revision to VA shunt due to long-term abdominal distension complicated by neonatal necrotizing enterocolitis and CSF overproduction after VP shunting.
\end{abstract}

Key Words: Cerebrospinal fluid; Hydrocephalus; Necrotizing enterocolitis; Ventriculoperitoneal shunt

\section{INTRODUCTION}

A cerebrospinal fluid (CSF) shunt is the most common treatment known to date in the treatment of hydrocephalus. Several operational techniques were developed to divert CSF from the ventricular system to other spaces in the human body, such as the peritoneal cavity, the pleural cavity, and the right atrium, which also have CSF absorptive capabilities ${ }^{10}$. Among them, a ventriculoperitoneal (VP) shunt is one of the most commonly favored procedures for CSF diversion ${ }^{12)}$.

However, when the patient presents with abdominal CSF absorption abnormalities or infections, a VP shunt becomes unsuitable and an alternative site for CSF drainage is required. One alternative to VP shunting is ventriculoatrial (VA) shunting, but VA shunting is still rarely used as a first-line treatment due to cardio pulmonary and renal complications as well as surgeon's preferences and bias ${ }^{7}$. We present a rare case where we first performed VP shunting and later changed to a VA shunt because of long-term abdominal distension.

\section{CASE REPORT}

One of 2 twin girls, born $946 \mathrm{~g}$ at 26 weeks, was delivered by caesarean section. Brain ultrasonography was conducted immediately after birth and she was diagnosed with grade II germinal matrix hemorrhage with intraventricular hemorrhage (Fig. 1A). She also underwent an ileostomy surgery for neonatal necrotizing enterocolitis 10 days after birth. Post-hemorrhagic hydrocephalus was identified in the brain computed tomography (CT) scan, which was conducted around the $7^{\text {th }}$ week after birth (Fig. 1B). Thereafter, she underwent several external ventricular drainage operations for post-hemorrhagic hydrocephalus and VP shunt surgery at 6 months of age (Fig. 1C). However, several months of vomiting and constipation ensued after the 

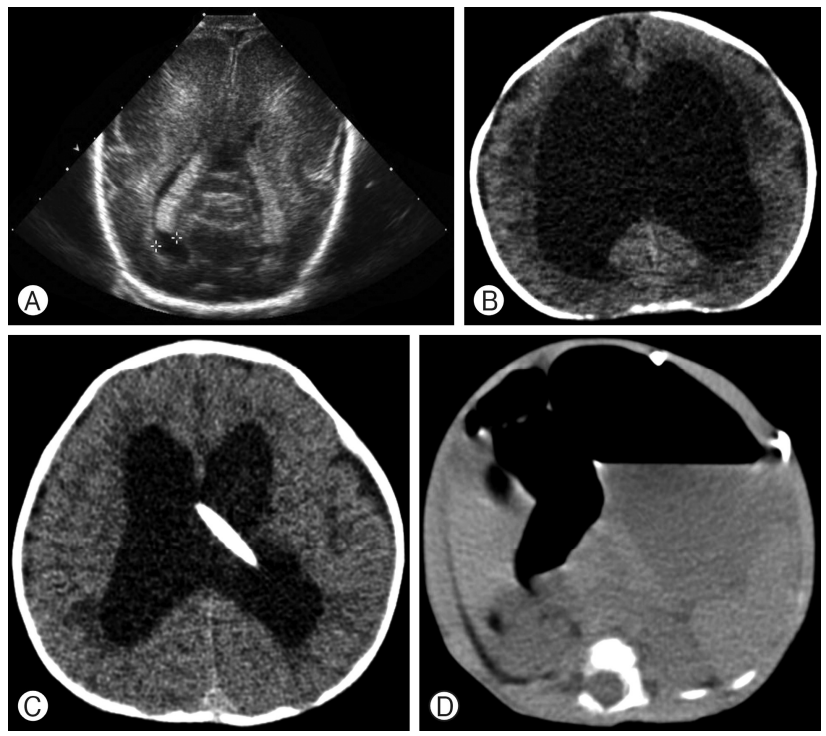

Fig. 1. (A) Postnatal brain ultrasonography showing intraventricular hemorrhage. (B) Ventricular enlargement after intraventricular hemorrhage 7 weeks after birth detected by brain computed tomography (CT) as traces of right frontal hemorrhage. (C) Brain CT demonstrates marked decrease in ventricular size after shunt procedure at 7 months after birth. (D) Abdominal CT scan image shows severe fluid accumulation and distal shunt catheter in the peritoneal cavity at 8 months of age.

VP shunt surgery, and the abdominal distension worsened. At 8 months of age, a large amount of fluid collection within the peritoneal cavity was confirmed by an abdominal CT scan (Fig. 1D)

The patient was 11 months old when she first came to our hospital with a VP shunt for the moderately large lateral ventricles without choroid plexus hypertrophy seen on brain magnetic resonance imaging (MRI) (Fig. 2A). The abdominal distension continued, and ventricular dilatation progressed (Fig. 2B). CSF drained from the distal VP shunt catheter was poorly absorbed within the peritoneal cavity. At 27 months of age, the second abdominal CT scan again revealed a large amount of fluid collection within the peritoneal cavity (Fig. 2C). At 29 months of age, distal abdominal catheter externalization was temporarily performed (Fig. 2D). Twenty to $30 \mathrm{~mL} / \mathrm{kg}$ of CSF per day was drained from 5 to $10 \mathrm{~cm}$ above the tragus point after distal abdominal catheter externalization, so CSF overproduction was suspected. Abdominal distension was still not resolved 2 weeks later even after the distal abdominal catheter externalization. VA shunting was performed with a Medtronic PS Medical, Inc. (Goleta, CA, USA) regular low pre- ssure valve. During insertion of the VA shunt, it was confirmed that the distal catheter tip was in the cavoatrial junction by intraoperative fluoroscopic imaging (Fig. 3A). The patient was discharged with improved abdominal distension and slightly smaller lateral ventricles (Fig. 3B). She was followed up for 3 years without any complications (Fig. 3C).
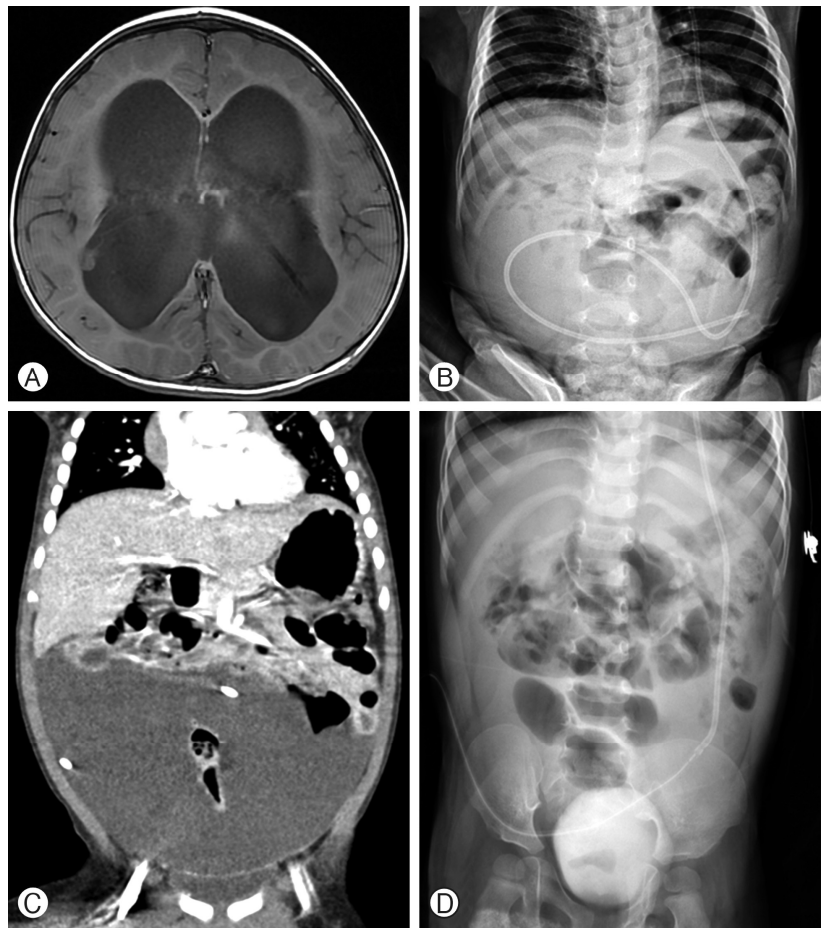

Fig. 2. (A) The magnetic resonance imaging performed at 11 months of age during the first visit to our hospital shows moderately large lateral ventricles without choroid plexus hypertrophy. (B) Abdominal distension is persistent in this abdominal X-ray. (C) Abdominal computed tomography scan image shows severe fluid accumulation and distal shunt catheter in peritoneal cavity. (D) Post-shunt externalization abdominal X-ray showing decreased abdominal distension.

\section{DISCUSSION}

A CSF shunt is one of the standard treatments for hydrocephalus and is required for $12 \%$ to $14 \%$ of patients with severe post-hemorrhagic hydrocephalus ${ }^{1,2,5)}$. Treatment methods for hydrocephalus aside from VP shunting include VA shunting, third ventriculostomy, choroid plexotomy, and choroidal artery embolization ${ }^{6,13)}$. Although CSF shunt placement is the most com- mon procedure performed by pediatric neurosurgeons, shunts remain among the most failure-prone implantation devices in modern medical practice, with failure rates of $30 \%$ to $40 \%$ at 1 year and approximately $50 \%$ at 2 years in pediatric patients $^{3)}$.

The most classical method of treatment for patients with hydrocephalus is the placement of a VP shunt where a distal catheter is placed into the peritoneal space, and it is important to recognize that intra-abdominal pressure can be one of the major determinants of effective shunt function. In such patients, constipation can result in significantly elevated intra-abdominal pressure that decreases CSF outflow through a distal catheter, resulting in shunt failure ${ }^{9)}$. Surgery due to abdominal problems 

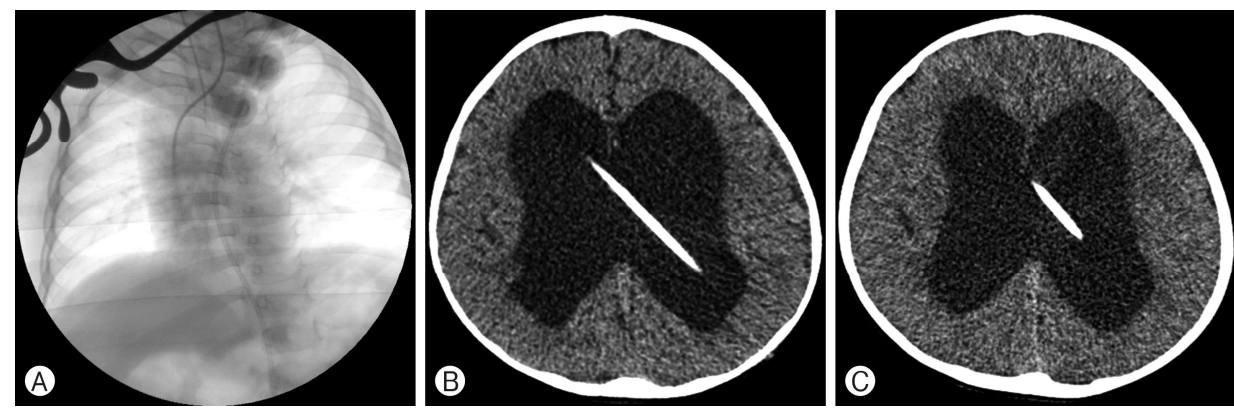

Fig. 3. (A) Intraoperative chest fluorography shows the tip of the distal catheter is well placed in the right atrium after ventriculoatrial shunt. (B) The immediate postoperative brain computed tomography (CT) shows slightly decreased large lateral ventricles and proximal shunt catheter. (C) The brain CT at 3 years postoperation shows smaller lateral ventricles and proximal shunt catheter.

such as necrotizing enterocolitis can decrease intra-abdominal absorption and result in shunt malfunction ${ }^{11)}$. In addition, abdominal distension from overproduction of CSF from choroid plexus hypertrophy has also been reported to affect shunt function ${ }^{6)}$.

VA shunts channel CSF from the ventricle of the brain into the right atrium of the heart. Although it is rarely considered as a first-line treatment because of cardiopulmonary and renal complications, VA shunting is a valid alternative in patients who have experienced multiple previous intra-abdominal VP shunt failures or had contraindications to abdominal catheter placement ${ }^{14)}$. Recently, there are some reports that show no significant differences in mortality between patients with VP shunts and VA shunts, so in circumstances where it is difficult to perform a VP shunt, a VA shunt may be considered as an alternative $^{11)}$.

When an intra-abdominal infection or abdominal surgery leads to an absolute decrease in CSF absorption, or when abdominal CSF absorption decreases due to absolute CSF overproduction especially due to choroid plexus hypertrophy, there is a high risk of VP shunt failure. In these cases, VA shunts should be considered rather than VP shunts ${ }^{6,8)}$. Despite this, when CSF absorption is absolutely or relatively decreased in the abdominal cavity, the current opinion is that VA shunts should be recommended only after experiencing repeated VP shunt failures since VA shunts may yield serious complications ${ }^{3,4,10)}$.

However, it is the opinion of the authors of this investigation that in patients who demonstrate both CSF overproduction and a decrease in CSF absorption, a VA shunt should be strongly considered over a VP shunt. In particular, this may be especially applicable for patients who experience long-term abdominal distension from VP shunt failure when repeated VP shunts are highly likely to fail. Therefore, prompt VA shunts should be provided as the first-line treatment modality.

\section{CONCLUSION}

Although VA shunting is not routinely performed as the first-line treatment method due to many serious complications, it may be an alternative treatment modality if the VP shunt procedure is unable to be sustained due to multiple causes, such as long-term abdominal distension from CSF overproduc- tion and poor absorption.

\section{CONFLICTS OF INTEREST}

No potential conflict of interest relevant to this article was reported.

\section{REFERENCES}

1. Adams-Chapman I, Hansen NI, Stoll BJ, Higgins R: Neurodevelopmental outcome of extremely low birth weight infants with posthemorrhagic hydrocephalus requiring shunt insertion. Pediatrics 121:e1167-e1177, 2008

2. Brouwer A, Groenendaal F, van Haastert IL, Rademaker K, Hanlo P, de Vries L: Neurodevelopmental outcome of preterm infants with severe intraventricular hemorrhage and therapy for post-hemorrhagic ventricular dilatation. J Pediatr 152:648-654, 2008

3. Hanak BW, Bonow RH, Harris CA, Browd SR: Cerebrospinal fluid shunting complications in children. Pediatr Neurosurg 52: 381-400, 2017

4. Hung AL, Vivas-Buitrago T, Adam A, Lu J, Robison J, Elder $\mathrm{BD}$, et al.: Ventriculoatrial versus ventriculoperitoneal shunt complications in idiopathic normal pressure hydrocephalus. Clin Neurol Neurosurg 157:1-6, 2017

5. Kahle KT, Kulkarni AV, Limbrick DD, Jr., Warf BC: Hydrocephalus in children. Lancet 387:788-799, 2016

6. Li D, Shokuhfar T, Pantalone J, Rothstein B, Alden TD, Shaibani A, et al.: Choroidal artery embolization in the management of cerebrospinal fluid overproduction: Case report and review of the literature. J Neurosurg Pediatr [epub ahead of print Mar 22, 2019. doi: 10.3171/2019.1.PEDS18519]

7. Liu A, Sankey EW, Jusué-Torres I, Patel MA, Elder BD, Goodwin $\mathrm{CR}$, et al.: Clinical outcomes after ventriculoatrial shun- 
ting for idiopathic normal pressure hydrocephalus. Clin Neurol Neurosurg 143:34-38, 2016

8. McGovern RA, Kelly KM, Chan AK, Morrissey NJ, McKhann GM, 2nd: Should ventriculoatrial shunting be the procedure of choice for normal-pressure hydrocephalus? J Neurosurg 120 : 1458-1464, 2014

9. Muzumdar D, Ventureyra EC: Transient ventriculoperitoneal shunt malfunction after chronic constipation: Case report and review of literature. Childs Nerv Syst 23:455-458, 2007

10. Niu H, Wu H, Luo W, Wang K, Zhao L, Wang Y: Ventriculoatrial shunt as a feasible regimen for certain patients of hydrocephalus: clinical features and surgical management. Acta Neurol Belg [epub ahead of print, 2019. doi: 10.1007/s13760-019-01180-w]

11. Pierro A, Manalang LR, May PL, Cooke RW, Cudmore RE, Lloyd DA: Necrotizing enterocolitis complicating the manage- ment of posthemorrhagic hydrocephalus. J Pediatr Surg 28:982985, 1993

12. Reddy GK, Bollam P, Shi R, Guthikonda B, Nanda A: Management of adult hydrocephalus with ventriculoperitoneal shunts: long-term single-institution experience. Neurosurgery 69:774780, 2011

13. Valdez Sandoval P, Hernández Rosales P, Quiñones Hernández DG, Chavana Naranjo EA, García Navarro V: Intraventricular hemorrhage and posthemorrhagic hydrocephalus in preterm infants: Diagnosis, classification, and treatment options. Childs Nerv Syst 35:917-927, 2019

14. Yavuz C, Demirtas S, Caliskan A, Kamasak K, Karahan O, Guclu $\mathrm{O}$, et al.: Reasons, procedures, and outcomes in ventriculoatrial shunts: A single-center experience. Surg Neurol Int [epub ahead of print, 2013. doi: 10.4103/2152-7806.106284] 